\title{
Determination of the Glass Transition Temperature of Freestanding and Supported Azo-Polymer Thin Films by Thermal Assisted Atomic Force Microscopy
}

\author{
Elena Chernykh $^{1}$, Sergey Kharintsev ${ }^{1,2,}$, Alexandr Fishman ${ }^{1}$, Alexander Alekseev ${ }^{3,4}$, Myakzuym Salakhov ${ }^{1,2}$ \\ ${ }^{1}$ Department of Optics and Nanophotonics, Institute of Physics, Kazan Federal University, Kremlevskaya, 16, Kazan, 420008, Russia \\ ${ }^{2}$ Institute of Perspective Technologies, Tatarstan Academy of Sciences, Baumana, 20, Kazan, 420111, Russia \\ ${ }^{3}$ National Laboratory Astana, Nazarbayev University, Kabanbay batyr ave., 53, Astana, 010000, Kazakhstan \\ ${ }^{4}$ CSF ECB\&MST, National Research University MIET, 124498 Moscow, Russia
}

\begin{abstract}
In this paper we introduce and apply the method for determination of the glass transition temperature of the sub-100 $\mathrm{nm}$ thick freestanding and supported polymer films based on thermally assisted atomic force microscopy (AFM). In proposed approach changes of the phase of an oscillating AFM cantilever are used to determine glass transition temperature. An anomalous decrease of the glass transition temperature for both free-standing and supported azobenzene-functionalized polymer thin films is shown.
\end{abstract}

\section{Introduction}

Azobenzene-functionalized polymers are one of the most promising materials in polymer photonics and organic electronics. Due to trans-to-cis photoisomerization, azochromophores found applications in formation of surface relief gratings and study of nonlinear optical effects. ${ }^{1-4}$ These effects are widely used in practice, for example for optical switching and storage, ${ }^{5-6}$ light filtering ${ }^{7-10}$ and frequency conversion. ${ }^{11}$ In particular, bottlenecks of optical near-field memory based on azo-polymers are cycling endurance, data retention, and thermal stability. Photoinduced alignment of the azo-dyes in glassy polymers is affected by the thickness of a polymer thin film, since the glass transition temperature $T_{\mathrm{g}}$ critically drops when the film thickness decreases. ${ }^{12}$ In addition, $T_{\mathrm{g}}$ is influenced by the interface interaction and, therefore, type of a substrate plays role as well. ${ }^{13,14}$

The integrity and thermal stability of the oriented state of polymer thin films are greatly sensitive to the glass transition temperature, at which an intrinsic structure of the polymer changes from a hard disordered solid to a rubber-like bulk. In turn, this influences on the increased mobility of a surface layer that underlies the most observable photo-induced effects in the azopolymers. The determination of the glass transition temperature has been the subject of through studies over last 50 years. Nowadays, there are many methods for determining the glass transition temperature of bulk amorphous and liquid crystalline polymers such as differential scanning calorimetry ${ }^{15}$, dynamic mechanical analysis $^{16}$, Raman scattering ${ }^{17}$, Brillouin scattering ${ }^{18}$ and others. However, most of these methods have a low sensitivity to the sub-100 nm thick polymer films. Atomic force microscopy is a promising technique, which provides information about glass transitions through a mechanical behavior of the AFM cantilever. In this paper, we determine the glass transition temperature of freestanding and supported azo-polymer films using thermal assisted atomic force microscopy.

\section{Experimental section}

Herein, we study thin films of epoxy-based oligomers containing hydroxyl groups with covalently attached nitroazobenzene chromophores, further it is referred to as CFAO. A chemical structure of this molecule is given in Fig. 1(a). The solution was prepared by dissolving 5 wt. \% CFAO compound in $1 \mathrm{ml}$ cyclohexanone. Polymer films with different thicknesses in the range of 5-500 nm were fabricated. The thin films were produced by spin coating $1 \mu \mathrm{l}$ CFAO solution onto a glass substrate at a rotation rate of $10000 \mathrm{rpm}$ for $2 \mathrm{~min}$. Freestanding films were prepared by centrifugation on a $\mathrm{KBr}$ substrate. Then substrate was dissolved in distilled water and after that thin polymer films were picked up with a mesh copper grids as shown in Fig. 1(b). The film thickness was determined by atomic force microscopy in contact mode. In order to minimize errors in determining the film thickness, we have built the dependence of the depth of lithographically produced "grooves" on applied force of the AFM cantilever. The film thickness was defined as a value that meets a plateau on this curve (see Fig.1(c)). 


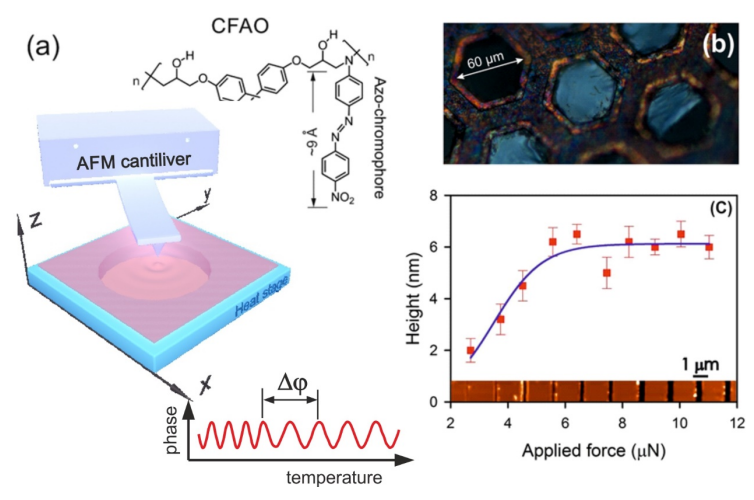

Fig. 1. a) experimental setup and chemical formula of CFAO; b) optical image of the freestanding CFAO film; c) plot of the groove depth on applied contact force.

The phase measurements were performed with an atomic force microscope Ntegra Prima (NT-MDT) in tapping mode. We used silicon AFM cantilever with the curvature radius of $10 \mathrm{~nm}$, the resonant frequency of 150 $\mathrm{kHz}$ and the force constant of $5 \mathrm{~N} / \mathrm{m}$ (NSG01, NTMDT). Polymer films were heated with the thermo stage (SU045NTF, NT-MDT) with an accuracy of $0.05{ }^{\circ} \mathrm{C}$ within the range from room temperature to $150{ }^{\circ} \mathrm{C}$. A schematic drawing of the experiment is given in Fig. 1(a). In our approach, the atomic force microscope was used as a dynamic mechanical analyzer of the AFM cantilever phase shift during in-situ heating/cooling at a rate of $5^{\circ} \mathrm{C} / \mathrm{min}$ (Fig. 1(a)).

\section{Results and discussion}

Due to the fact that the phase of the cantilever oscillations is sensitive to the surface properties, we can use this parameter to monitor the glass transition of polymer films. It is known that cantilever phase shift $\varphi$ is determined by energy dissipated during tip-sample interaction: $\sin \varphi \sim \mathrm{E}_{\mathrm{dis}}$, where $E_{\mathrm{dis}}$ is energy dissipated during one period of oscillation. ${ }^{19}$

The phase of the cantilever oscillations is influenced by viscoelasticity, friction, adhesion etc. which leads to energy dissipation. This can be attributed to the loss of energy of the cantilever, which is associated with a change in the Gibbs free energy of the polymer: $\Delta G=\mathbb{A} F \boldsymbol{S}$. At the glass transition temperature, changes in enthalpy $\Delta H$ and entropy $\Delta S$ of the CFAO thin films are changed and it can lead to reversible jumps of the AFM phase during heating (red curve) and cooling (blue curve). ${ }^{20}$ For stable and reliable AFM measurements with in-situ heating/cooling, we heat or cool the polymer step-by-step by a value of $20^{\circ} \mathrm{C}$ at a linear thermal rate of $5{ }^{\circ} \mathrm{C} / \mathrm{min}$. This stepwise regime permits the sample to be in a near-equilibrium state. Using this approach, $T_{\mathrm{g}}$ can be defined as the temperature at which the jump on phase curve occurs during heating/cooling, as shown in Fig. 2(a).
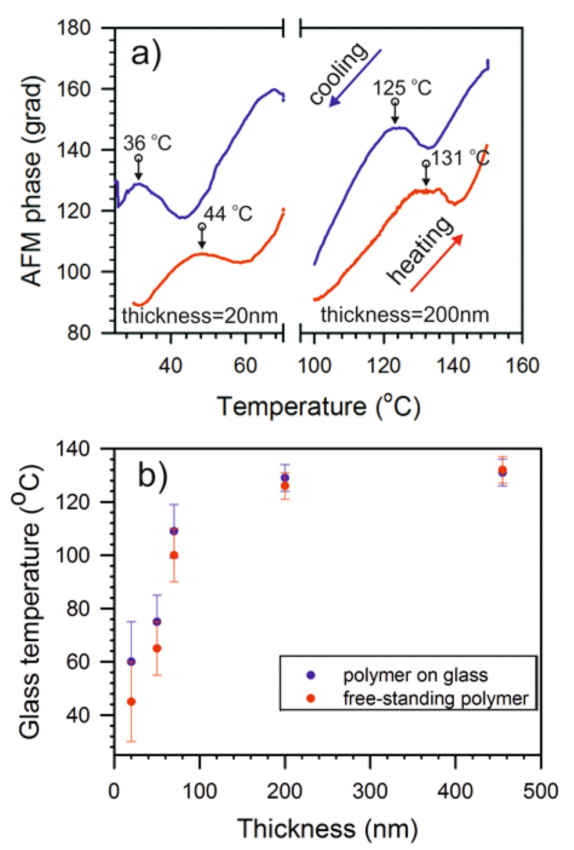

Fig. 2. A plot of the AFM phase vs. temperature of the CFAO thin film with thickness of $20 \mathrm{~nm}$ and $200 \mathrm{~nm}$ (a), a dependence of the glass transition temperature on the thickness of freestanding and supported CFAO films.

This figure shows results of measurements of the glass transition temperature for both $20 \mathrm{~nm}$ and $200 \mathrm{~nm}$ thick CFAO films. The $T_{\mathrm{g}}$ value for the bulk film coincides with the results obtained with other methods and is equal to $\sim 130{ }^{\circ} \mathrm{C}$. For a thiner CFAO film we observe the decrease of $T_{\mathrm{g}}$ down to roughly $40{ }^{\circ} \mathrm{C}$. The depression of the glass transition temperature with the decrease of film thickness was shown in numerous studies. ${ }^{13,17,21}$ Thus, our results are in agreement with earlier published ones. Fig. 2(b) demonstrates the influence of thickness on $T_{\mathrm{g}}$ for both free-standing and supported CFAO thin films. As follows from this figure, the $T_{\mathrm{g}}$ behaviours for both freestanding and supported CFAO thin films are close to each other. It means that a role of the substrate in our case is negligable. This was experimentally confirmed by a poor wettability of the CFAO film. Indeed, very thin films with the thcikness of less than $7 \mathrm{~nm}$ exhibit a ragged stucture on the subsrate surface.

\section{Conclusions}

The glass transition temperature of CFAO thin films can be determined by using thermal assisted atomic force microscopy in tapping mode. The applied method is based on high sensitivity of the oscillating cantilever phase to changes of the surface properties. This method gives reliable and robust estimations for the glass transition temperature for very thin films with the thickness of less than $20 \mathrm{~nm}$.

\section{Acknowledgment}

This work was financially supported by the Russian Foundation for Basic Research (No. 15-42-02339). This 
work was done using equipment of Federal Center of Shared Equipment of Kazan Federal University.

\section{References}

1. N.A. Nikonorova, M.Yu. Balakina, O.D. Fominykh, M.S. Pudovkin, T.A. Vakhonina, R. Diaz-Calleja, A.V. Yakimansky, Chem. Phys. Lett. 522, 114 (2012)

2. Photo-reactive organic thin films; Z. Sekkat, W. Knoll. Eds; Academic press: Elsevier Science, USA, 2002.

3. Z. Sekkat, M. Buchel, H. Orendi, H. Menzel, W. Knoll, Chem. Phys. Lett. 220, 497 (1994).

4. A. Natansohn, P. Rochon, Chem. Rev. 102, 4139 (2002)

5. A. Natansohn, P. Rochon, M. Pezolet, P. Audet, D. Brown, S., Macromolec. 27, 2580 (1994)

6. W.Shi, Yu. J. Ding, X. Mu, Appl. Phys. Lett. 79, 3749 (2001)

7. Y. Wang, O.Y.-H. Tai, C.H. Wang, J. Chem. Phys. 2005. 123, 704 (2005)

8. M.S. Ho, A. Natansohn, Macromolec. 28, 6124 (1995)

9. S.S. Kharintsev, A.I. Fishman, S.G. Kazarian, I.R. Gabitov, M.Kh Salakhov, ACS Photonics. 1, 1025 (2014)

10. S.S. Kharintsev, A.I. Fishman, S.K. Saikin, S.G. Kazarian, Nanoscale 8, 19867 (2016).

11. C. Fiorini, F. Charra, J.-M. Nunzi, P. Raimond, J. Opt. Soc. Am. B. 14, 1984 (1997)

12. J.L. Keddie, R.A.L. Jones, R.A. Cory, Faraday Discuss. 98, 219 (1994)

13. D.S. Fryer, P.F. Nealey, J.J. de Pablo, Macromolec. 33, 6439 (2000)

14. J.A Torres, P.F. Nealey, J.J. de Pablo, Phys. Rev. Lett. 85, 3221 (2000)

15. E. Dargent, C. Cabot, J.M. Saiter, J. Bayard, J. Grenet, Jour. of Therm. Analys. 47, 887 (1996)

16. B. Twombly, D.D. Shepard, Instrum. sci. and technolog. 22, 259 (1994)

17. H. Liem, J. Cabanillas-Gonzalez, P. Etchegoin, D.D.C Bradley, Journ. of Phys.: Cond. Matt. 16, 721(2004)

18. J.A. Forrest, K. Dalnoki-Veress, J.R. Dutcher, A.C. Rowat, J.R. Stevens, Disordered materials and interfaces. 407, 131 (1996)

19. J. P. Cleveland, B. Anczykowski, A.E. Schmid, V.B. Elings, Appl. Phys. Lett. 72, 2613-2615 (1998).

20. M. Meincken, L.J. Balk, R.D. Sanderson, Surf. Interface Anal. 35, 1034 (2003)

21. D. Liu, R.O. Orozco, T. Wang, Phys. Rev. E. 88, 22601 (2013) 